\title{
Myocardial Injury at Early Stage and Its Association with Death Risk of Patients with COVID-19: A Hospital-Based Prospective Case-Cohort Study
}

\section{Lin Fu}

Anhui Medical University

Xiu-Yong Li

Second People's Hospital of Fuyang City

Jun Fei

Anhui Medical University

Ying Xiang

Anhui Medical University

\section{Hui-Xian Xiang}

Anhui Medical University

\section{Meng-Die Li}

Anhui Medical University

\section{Fang-Fang Liu}

Anhui Medical University

\section{Ying Li}

Huazhong University of Science and Technology

Hui Zhao

Anhui Medical University

De-Xiang Xu ( $\sim$ xudex@126.com)

Anhui Medical University https://orcid.org/0000-0002-5126-4335

\section{Research article}

Keywords: Severe acute respiratory syndrome coronavirus-2 (SARS-CoV-2), Coronavirus disease 2019 (COVID-19), Myocardial injury, Prognosis

Posted Date: June 16th, 2020

DOI: https://doi.org/10.21203/rs.3.rs-34902/v1

License: (a) (i) This work is licensed under a Creative Commons Attribution 4.0 International License.

Read Full License 


\section{Abstract}

Background: There are growing evidence demonstrating that coronavirus disease 2019 (COVID-19) is companied by acute myocardial injury. However, the association of SARS-CoV-2-induced myocardial injury with death risk of COVID-19 is unclear.

Methods: This prospective case-cohort study analyzed 355 COVID-19 patients from two hospitals in different regions. Clinical and demographic information were collected. Myocardial injury was evaluated and its prognosis was followed up.

Results: Of 355 hospitalized patients with COVID-19, 213 were mild, 90 severe and 52 critically ill patients. On admission, 220 (62.0\%) patients were with myocardial injury. Myocardial injury was more popular in critically ill patients. Using multivariate logistic regression, male, older age and comorbidity with hypertension were three crucial independent risk factors predicting myocardial injury of COVID-19 patients. Among 220 COVID-19 patients with myocardial injury, 33 (15.0\%) died on mean 10.9 day after hospitalization. Mortality was increased among COVID-19 patients with myocardial injury ( $15.0 \% \mathrm{vs}$ $1.74 \%, R R=8.625, P<0.001)$. Follow-up study observed that at least one myocardial index of $21.3 \%$ patients remained abnormal 14 days after discharge.

Conclusion: Myocardial injury at early stage elevates mortality of COVID-19 patients. Male elderly patients with hypertension are more vulnerable to myocardial injury. SARS-CoV-2-induced myocardial injury has not completely recovered 14 days after discharge.

\section{Background}

Coronavirus Disease 2019 (COVID-19) is a newly recognized infectious disease caused by the newly discovered Severe Acute Respiratory Syndrome- Coronavirus-2 (SARS-CoV-2). COVID-19 firstly broke out in Wuhan city, Hubei province in China since December 2019 [1]. Now, it has been pandemic all over the world. Up to April 19th, China has cumulatively diagnosed 84225 cases and over 4642 deaths, while the number of cases in other countries is growing rapidly with a total of 2277363 confirmed cases and 157572 death cases [2]. American and Europe have gradually become the epicenter of the pandemic and the entire world is suffering great public health crisis from SARS-CoV-2, which is more severe than 2003 SARS disaster [3].

Some studies have found that SARS-CoV-2 transmitted through not only droplets or direct contact but also feces [4, 5]. Evidence pointing to the person-to-person transmission has occurred among close contacts in hospital and family [6]. As reported by other researchers and our team found that patients with COVID-19 present primarily with fever, diarrhea, fatigue, dry cough, lymphopenia and radiographic evidence of pneumonia [7-9]. The Chinese Center for Disease Control and Prevention recently revealed that the death risk of mild COVID-19 patients was relatively low in a large Chinese population-based study, the fatality rate was significantly elevated among critical ill COVID-19 cases [10]. Previous studies found that SARS-CoV-2 mainly evoked severe acute respiratory syndrome. More and more researches 
revealed that most cases were prone to suffer multiple organ injuries, such as immune system disorder, acute kidney injure and even liver dysfunction [11-13]. Nevertheless, the clinical characteristics of myocardial injury caused by SARS-CoV-2 are rarely described. In addition, the clinical significance of SARS-CoV-2-induced myocardial injury and its prognosis are still unclear.

The aim of this study was to investigate SARS-CoV-2-induced myocardial injury, its association with death risk of COVID-19 and prognosis after discharge. Our results suggested that male elderly COVID-19 patients with hypertension were more vulnerable to myocardial injury. We found that myocardial injury at early stage increased the risk of death among COVID-19 patients. This study first provides evidence that SARS-CoV-2 induced myocardial injury has not completely recovered 14 days after discharge.

\section{Methods}

Study design and participants

In the present study, 200 patients with COVID-19 were recruited in Union Hospital of Huazhong University of Science and Technology in Wuhan city from January 1 to January 30, 2020. Another 155 patients with COVID-19 were recruited from the Second People's Hospital of Fuyang City in Anhui province. Union Hospital of Huazhong University of Science and Technology and Second People's Hospital of Fuyang City were assigned responsibility for the treatment of patients with COVID-19 by the Anhui government and Wuhan government. All patients were laboratory confirmed positive of SARS-CoV-2 injection by RT-PCR on pharyngeal swab specimens. Diagnosis of COVID-19 was based on the New Coronavirus Pneumonia Prevention and Control Program (6th edition) published by the National Health Commission of China. There was no death case with COVID-19 in the Second People's Hospital of Fuyang City. All patients were discharged. At last, 150 cured cases were performed follow-up examinations 14 days after discharge in the Second People's Hospital of Fuyang City, biochemical indexes and blood routine were detected among COVID-19 patients again. This study was approved by the institutional ethics board of Union Hospital of Huazhong University of Science and Technology, and Second People's Hospital of Fuyang City. All COVID-19 patients were eligible in this study. Oral consent was obtained from patients or patients' next of kin.

\section{Data collection}

The medical record of each COVID-19 patient was collected. Patient's data including demographics, comorbidities (chronic obstructive pulmonary disease, hepatic disease, cardiovascular disease, hypertension, diabetes and other disease), patient's signs and symptoms, and laboratory test results were also collected. The date of onset and outcomes were recorded.

\section{Laboratory testing}

Patient's pharyngeal swab specimens were measured. Real-time RT-PCR was used to detect viral nucleic acid using a COVID-19 nucleic acid detection kit following experimental instructions (Shanghai bio-germ 
Medical Technology Co Ltd). Aspartate aminotransferase (AST), creatine kinase (CK), creatine kinase isoenzyme (CKMB), lactate dehydrogenase (LDH), oxygenation index (PaO2/FiO2), C-reactive protein (CRP),were examined on admission. All laboratory tests were measured by the clinical laboratory.

Statistical analysis

All statistical analyses were performed using SPSS19.0 software. Categorical variables were expressed with frequencies and percentages. Continuous variables were shown using median and mean values. All categorical variables were compared for the study outcome by using the Fisher exact test or $\chi^{2}$ test, and continuous variables were compared via the t test or the Mann-Whitney $\mathrm{U}$ test, as appropriate. Logistic regression analysis between different parameter and myocardial injure as well as death risk and myocardial injure were performed. Statistical significance was determined at $P \otimes 0.05$.

\section{Results}

\section{Demographic and clinical characteristics}

All 355 COVID-19 patients' clinical information was collected and evaluated. As shown in Table 1, common case, defined as oxygenation index higher than 300, was 213 (60.0\%). For severe case, whose oxygenation index was from 200 to 300, was 90 (25.4\%). For critically ill case, whose oxygenation index was lower than 200, accounted for $14.6 \%$ (Table 1). Moreover, the demographic characteristics were then analyzed. As shown in Table 2, 162 (45.6\%) were female and 193 (54.4\%) were male. There were 96 patients younger than 39 years old, 144 patients aged between 40 and 59, and 115 patients older than 60 years old. Of 355 patients with COVID-19, 230 (64.8\%) patients had hypertension, 208 (58.6\%) patients had diabetes and $20(5.63 \%)$ had chronic heart disease.

\section{Association of myocardial injury with the severity of COVID-19 patients}

The association between myocardial injury and the severity of COVID-19 was evaluated in patients. Myocardial injury indexes, including CK, CKMB, LDH and AST,were analyzed. As shown in Table 1, the level of CK were higher in critical ill patients than those of mild and severe patients. The number of CKMB-positive patients were more in critical ill patients than those of mild patients. The levels of LDH and AST were the lowest in the mild patients with COVID-19. Moreover, the levels of LDH and AST were higher in the critical ill patients than those of in severe patients. Myocardial injury was defined as any of myocardial functional indexes beyond normal range. The results indicated that 114 (53.5\%) COVID-19 patients were with myocardial injury at early stage in mild patients. $61(67.8 \%)$ patients with myocardial injury were in severe patients and $45(86.5 \%)$ patients with myocardial injury were in critically ill patients. Furthermore, the associations between oxygenation index and myocardial injury markers were analyzed. As shown in Supplemental Table 1, no association between CK and CKMB with oxygenation index was observed, there was a negative association between AST $(r=-0.249, P=0.001)$ and LDH $(r=-0.431$, $P \otimes 0.001)$ with oxygenation index among COVID-19 patients. Additionally, the associations between inflammatory cytokine and myocardial injury markers were analyzed. The results indicated that CRP was 
positively correlated with AST $(r=0.241, P=0.004), \mathrm{LDH}(r=0.457, P ख 0.001)$ and CK $(r=-0.198, P=0.018)$ (Supplemental Table 1).

\section{Male elderly COVID-19 patients with hypertension are more vulnerable to myocardial injury}

The effects of demographic characteristics on myocardial injury markers were analyzed. As shown in Table 2, the level of CK were higher in males than in females. There was no different of CKMB, LDH and AST between females and males. Further analysis showed that CK, CKMB, LDH and AST were lower in patients younger than 39 years old than those of older patients. Moreover, we found that CK, CKMB, LDH and AST were higher in patients older than 60 than those between 40 from 59 years old (Table 2). The effects of comorbidities on myocardial functional indexes were then analyzed. As shown in Table 2 , CKMB-positive patients were more in COVID-19 patients with hypertension than those without hypertension. Besides, the level of CK was elevated in COVID-19 patients with diabetes compared with those without diabetes. LDH and AST were increased in COVID-19 patients with chronic heart disease compared with those without heart disease. In addition, the risk factors of myocardial injury were analyzed using multivariable logistic regression among COVID-19 patients. As shown in Table 3, the $O R$ of male gender was 2.012 (95\% Cl: $1.125,3.599)$, the $O R$ of age was $1.434(95 \% \mathrm{Cl}: 1.041,1.976)$ and the $O R$ of hypertension was $3.393(95 \% \mathrm{Cl}: 1.441,7.989)$ for myocardial injury, respectively.

\section{Myocardial injury at early stage elevates death risk of COVID-19 patients}

The effects of myocardial injury at the early stage on death risk are presented in Table 4. Among 220 COVID-19 patients with myocardial injury, $10.4 \%$ were died. The fatality rate was higher among COVID-19 patients with myocardial injury than those without myocardial injury $(15.0 \%$ vs $1.74 \% ; R R=8.625,95 \% \mathrm{Cl}$ : 2.107, 35.305; $P<0.001)$.

\section{Myocardial markers remain abnormal 14 days after discharge}

The recovery of myocardial injury markers was investigated in every patient with COVID-19. Myocardial markers were compared between on admission and 14 days after discharge in the Second People's Hospital of Fuyang City. As shown in Table 5, there was no significant difference in the levels of CK, CKMB and AST among COVID-19 patients between on admission and after discharge, whereas LDH was decreased on 14 days after discharge than on admission. On admission, $5(3.3 \%)$ cases with CK, 9 (6.1\%) cases with CKMB, 56 (38.1\%) cases with LDH and 19 (12.3\%) cases with AST were above the normal range. In all, there was 68 (44.2\%) COVID-19 patients with myocardial injury. The prognosis of COVID-19 patients' myocardial injury markers was followed up 14 days after discharge in the Second People's Hospital of Fuyang City. We found that 2 (1.33\%) patients with CKMB, 25 (16.7\%) patients with LDH and 25 (15.3\%) patients with AST remained above the normal range. Further analysis showed that 32 (21.3\%) patients with COVID-19 continuously accompanied with myocardial injury 14 days after discharge (Table 5).

\section{Discussion}


This study mainly investigated SARS-CoV-2-induced myocardial injury, its association with mortality and the prognosis 14 days after discharge. The major results of this study include: (1) Myocardial injury is more popular in the critically ill patients with COVID-19; (2) Male elderly COVID-19 patients with hypertension are more vulnerable to myocardial injury; (3) Myocardial injury at early stage elevates death risk of COVID-19 patients; (4) Myocardial injury markers of 21.3\% COVID-19 patients has not completely recovered 14 days after discharge.

More and more studies have demonstrated that COVID-19 patients were accompanied with multiple organ injuries, mainly including acute liver injury, acute kidney injury, respiratory failure and even lymphopenia [12-15]. In the present study, myocardial injury was evaluated through measuring biochemical indexes, such as CK, CKMB, LDH and AST. We found that four myocardial injury markers were higher in critically ill patients than those in mild and severe patients with COVID-19. Moreover, the cases of myocardial injury were more in critically ill patients than those in mild and severe patients with COVID-19. Myocardial injury was more popular in critically ill patients. These results provide evidence that myocardial injury at early stage is positively associated with the severity of COVID-19 patients.

The previous studies have revealed that older age patients have more severe symptoms and signs [12, 16]. In the present study, the effects of demographic characteristics on myocardial injury were analyzed. Although no difference of CKMB, LDH and AST was observed between females and males, CK was higher in males than those in females. Further analysis indicated that the levels of serum CK, LDH and AST were higher. In addition, the number of CKMB-positive cases was more in older patients than younger ones. Several reports indicated that comorbidities elevated the death risk and the severity of COVID-19 patients $[17,18]$. The present study found that $64.8 \%$ patients were with hypertension, $58.6 \%$ patients were with diabetes and $5.63 \%$ patients were with chronic heart disease. In order to investigate the influence of comorbidities on myocardial injury, the markers of myocardial injury were analyzed among COVID-19 patients. This study found that the number of CKMB-positive cases was more in patients with hypertension than those without hypertension. In addition, the level of serum CK was slightly increased in the patients with diabetes. The level of serum LDH was significantly higher in patients with chronic heart disease as compared with those without heart disease. These results indicate that male, older age and comorbidities may aggravate myocardial injury of COVID-19 patients. In order to further analyze the association between demographic characteristics with comorbidities and myocardial injury, the multivariate logistic regression analysis was performed. Our results indicated that male, older age, comorbidity with hypertension were three independent risk factors of myocardial injury. Generally speaking, male elderly COVID-19 patients with hypertension are more vulnerable to myocardial injury.

The effect of myocardial injury on the prognosis of COVID-19 patients is not yet clear. The association between myocardial injury and death risk was analyzed among COVID-19 patients. The present study found that the mortality was higher in COVID-19 patients with myocardial injury than those without myocardial injury. Myocardial injury on admission obviously elevates the death risk among COVID-19 patients. This is an urgent issue which is worthy of studying whether myocardial injury recovers during a short-period after discharge. In this work, 150 COVID-19 patients were tracked and markers of myocardial 
injury were detected. The rate of myocardial injury between on admission and 14 days after discharge were compared among COVID-19 patients from the Second People's Hospital of Fuyang City. Although no remarkably difference of the levels of serum CK, CKMB and AST was observed between on admission and 14 days after discharge, the level of serum LDH was obviously decreased on 14 days after discharge. In spite of the abnormal number of CKMB and LDH were decreased, $1.33 \%$ patients with CKMB and $16.7 \%$ patients with LDH were above the normal range. Our results indicate that myocardial injury markers of approximately one-five cases were abnormal 14 days after discharge. Therefore, whether SARS-CoV-2 causes continuous myocardial injury is needed to performed further follow-up research in the future clinical work.

The mechanism of which SARS-CoV-2 induces myocardial injury is scarcely clear. The previous study found that CRP was evidently increased in the critically ill patients [19,20]. CRP is an acute-phase protein in response to inflammatory cytokines after infections. High level of CRP partially reflects the severity of inflammation and evokes cytokine storm, which largely enhances vascular permeability and impairs organ function. Our results found that CRP was positively correlated with the levels of AST, LDH and CK, indicating that SARS-CoV-2-induced cytokine storm may be one of the mechanisms of myocardial injury. Both our research and other team found that SARS-CoV-2 injection reduced oxygenation index and caused respiratory function failure $[9,21]$ Continuous blood hypoxia induces acidosis and excess generation of reactive oxygen species, ultimately damaging myocardial cell. In the present study, we found that oxygenation index was negatively associated with AST and LDH among COVID-19 patients, suggesting that respiratory function failure may contribute, at least partially, to SARS-CoV-2-evoked myocardial injury. Increasing data demonstrate that angiotension converting enzyme (ACE)2, as a receptor for SARS-CoV-2, exerts a significant role in the pathogenesis of COVID-19 patients [22-24]. Recently, a report found that ACE2 was also expressed in cardiocytes [25]. Hence, these evidences don't exclude that SARS-CoV-2 evokes myocardial injury partially through directly damaging myocardial cells.

In summary, the present study mainly analyzed SARS-CoV-2-evoked myocardial injury among COVID-19 patients in two hospitals from different region. These results showed that SARS-CoV-2-induced myocardial injury was more general in critically ill patients. Furthermore, male elderly COVID-19 patients with hypertension were more vulnerable to myocardial injury. Our results firstly suggest that myocardial injury at early stage elevates death risk of COVID-19 patients. What's more, SARS-CoV-2-evoked myocardial injury has not completely recovered 14 days after discharge. Therefore, it is essential to further investigate whether SARS-CoV-2 results in a long-term myocardial injury.

\section{Abbreviations}

AST: Aspartate aminotransferase; COVID-19: Coronavirus disease 2019; CRP: C-reactive protein; CK: Creatine kinase; CKMB: Creatine kinase isoenzyme; LDH: Lactate dehydrogenase; $\mathrm{PaO2/FiO2:}$

Oxygenation index; SARS-CoV-2: Severe acute respiratory syndrome coronavirus-2.

\section{Declaration}




\section{Acknowledgments}

We greatly appreciate all doctors and nurses of respiratory and critical care medicine in the second People's Hospital of Fuyang City and Union Hospital of Huazhong University of Science and Technology for recruiting participators. Moreover, we also thank all patients and their families participated in this research.

\section{Availability of data and materials}

The datasets used and analysed are available from the corresponding author on reasonable request.

\section{Funding}

This study was supported by National Natural Science Foundation of China (grants number: 81630084) and National Natural Science Foundation Incubation Program of the Second Affiliated Hospital of Anhui Medical University (grant number: 2019GQFY06).

\section{Author contributions}

D.X.X., H.Z. designed research; L.F., X.Y.L., J.F., H.X.X., Y.X., M.D.L, F.F.L., and Y.L. conducted research; L.F., X.Y.L. and J.F. analyzed data; D.X.X. and L.F. wrote the paper; D.X.X. and L.F. had primary responsibility for final content. All authors read and approved the final manuscript.

\section{Competing interests}

The authors declare that they have no competing interests

\section{Consent for publication}

Not applicable.

\section{Ethics approval}

This study was approved by the institutional ethics board of Union Hospital of Huazhong University of Science and Technology, and Second People's Hospital of Fuyang City. All COVID-19 patients were eligible in this study. Oral consent was obtained from patients or patients' next of kin.

\section{Author details}

${ }^{1}$ Second Affiliated Hospital, Anhui Medical University, Hefei, Anhui Province, 230032, China; ${ }^{2}$ School of Public Health, Anhui Medical University, Hefei, Anhui Province, 230032, China; ${ }^{3}$ The second People's Hospital of Fuyang City, Fuyang, Anhui Province, 236015, China; ${ }^{4}$ Union Hospital, Huazhong University of Science and Technology, Wuhan, Hubei Province, 430040, China.

\section{References}


1. Li Q, Guan X, Wu P, Wang X, Zhou L, Tong Y, Ren R, Leung KSM, Lau EHY, Wong JY, et al. Early Transmission Dynamics in Wuhan, China, of Novel Coronavirus-Infected Pneumonia. N Engl J Med. 2020; 382(13): 1199-207.

2. World Health Organization. Coronavirus disease (COVID-19) Pandemic. https://www.who.int/emergencies/diseases/novel-coronavirus-2019.

3. World Health Organization, 2020c. WHO Director-General's opening remarks at the media briefing on COVID-19-13 March 2020. https://www.who.int/dg/speeches/ detail/who-director-general-s-openingremarks-at-the-mission-briefing-on-covid-19-13-march-2020.

4. Lai CC, Shih TP, Ko WC, Tang HJ, Hsueh PR. Severe acute respiratory syndrome coronavirus 2 (SARSCoV-2) and coronavirus disease-2019 (COVID-19): The epidemic and the challenges. Int J Antimicrob Agents. 2020; 55(3): 105924.

5. Yang X, Yu Y, Xu J, Shu H, Xia J, Liu H, Wu Y, Zhang L, Yu Z, Fang M, et al. Clinical course and outcomes of critically ill patients with SARS-CoV-2 pneumonia in Wuhan, China: a single-centered, retrospective, observational study. Lancet Respir Med. 2020; 8(5): 475-81.

6. Huang C, Wang Y, Li X, Ren L, Zhao J, Hu Y, Zhang L, Fan G, Xu J, Gu X, et al. Clinical features of patients infected with 2019 novel coronavirus in Wuhan, China. Lancet. 2020; 395(10223): 497-506.

7. Wu C, Chen X, Cai Y, Xia J, Zhou X, Xu S, Huang H, Zhang L, Zhou X, Du C, et al. Risk Factors Associated With Acute Respiratory Distress Syndrome and Death in Patients With Coronavirus Disease 2019 Pneumonia in Wuhan, China. JAMA Intern Med. 2020. doi: 10.1001/jamainternmed.2020.0994.

8. Fu L, Fei J, Xu S, Xiang HX, Xiang Y, Tan ZX, Li MD, Liu FF, Li Y, Han MF, et al. Influence factors of death risk among COVID-19 patients in Wuhan, China: a hospital-based case-cohort study. MedRxiv. 2020; 035329. doi: https://doi.org/10.1101/2020.03.13.20035329.

9. Fei J, Fu L, Li Y, Xiang HX, Xiang Y, Li MD, Liu FF, Xu DX, Zhao H. Reduction of lymphocyte at early stage elevates severity and death risk of COVID-19 patients: a hospital-based case-cohort study. MedRxiv. 2020; 050955. doi: https://doi.org/10.1101/2020.04.02.20050955.

10. Zhou F, Yu T, Du R, Fan G, Liu Y, Liu Z, Xiang J, Wang Y, Song B, Gu X, et al. Clinical course and risk factors for mortality of adult inpatients with COVID-19 in Wuhan, China: a retrospective cohort study. Lancet. 2020; 395 (10229): 1054-62.

11. Li G, Fan Y, Lai Y, Han T, Li Z, Zhou P, Pan P, Wang W, Hu D, Liu X, et al. Coronavirus infections and immune responses. J Med Virol. 2020; 92(4): 424-32.

12. Fu L, Fei J, Xu S, Xiang HX, Xiang Y, Tan ZX, Li MD, Liu FF, Li Y, Han MF, et al. Acute liver injury and its association with death risk of patients with COVID-19: a hospital-based prospective case-cohort study. MedRxiv. 2020; 050997. doi: https://doi.org/10.1101/2020.04.02.20050997.

13. Xu S, Fu L, Fei J, Xiang HX, Xiang Y, Tan ZX, Li MD, Liu FF, Li Y, Han MF, et al. Acute kidney injury at early stage as a negative prognostic indicator of patients with COVID-19: a hospital-based retrospective analysis. MedRxiv. 2020; 042408. doi: https://doi.org/10.1101/2020.03.24.20042408. 
14. Giamarellos-Bourboulis EJ, Netea MG, Rovina N, Akinosoglou K, Antoniadou A, Antonakos N, Damoraki G, Gkavogianni T, Adami ME, Katsaounou P, et al. Complex Immune Dysregulation in COVID-19 Patients with Severe Respiratory Failure. Cell Host Microbe. 2020. pii: S19313128(20)30236-5.

15. Spiezia L, Boscolo A, Poletto F, Cerruti L, Tiberio I, Campello E, Navalesi P, Simioni P. COVID-19Related Severe Hypercoagulability in Patients Admitted to Intensive Care Unit for Acute Respiratory Failure. Thromb Haemost. 2020. doi: 10.1055/s-0040-1710018.

16. Chen N, Zhou M, Dong X, Qu J, Gong F, Han Y, Qiu Y, Wang J, Liu Y, Wei Y, et al. Epidemiological and clinical characteristics of 99 cases of 2019 novel coronavirus pneumonia in Wuhan, China: a descriptive study. Lancet. 2020; 395(10223): 507-13.

17. Du Y, Tu L, Zhu P, Mu M, Wang R, Yang P, Wang X, Hu C, Ping R, Hu P, et al. Clinical Features of 85 Fatal Cases of COVID-19 from Wuhan: A Retrospective Observational Study. Am J Respir Crit Care Med. 2020. doi: $10.1164 / \mathrm{rccm}$.202003-05430C.

18. Cheng Y, Luo R, Wang K, Zhang M, Wang Z, Dong L, Li J, Yao Y, Ge S, Xu G. Kidney disease is associated with in-hospital death of patients with COVID-19. Kidney Int. 2020; 97(5): 829-38.

19. Xu B, Fan CY, Wang AL, Zou YL, Yu YH, He C, Xia WG, Zhang JX, Miao Q. Suppressed T cell-mediated immunity in patients with COVID-19: a clinical retrospective study in Wuhan, China. J Infect. 2020. pii: S0163-4453(20)30223-1.

20. Chen X, Zhao B, Qu Y, Chen Y, Xiong J, Feng Y, Men D, Huang Q, Liu Y, Yang B, et al. Detectable serum SARS-CoV-2 viral load (RNAaemia) is closely correlated with drastically elevated interleukin 6 (IL-6) level in critically ill COVID-19 patients. Clin Infect Dis. 2020. pii: ciaa449.

21. Giamarellos-Bourboulis EJ, Netea MG, Rovina N, Akinosoglou K, Antoniadou A, Antonakos N, Damoraki G, Gkavogianni T, Adami ME, Katsaounou P, et al. Complex Immune Dysregulation in COVID-19 Patients with Severe Respiratory Failure. Cell Host Microbe. 2020. pii: S19313128(20)30236-5.

22. Hoffmann M, Kleine-Weber H, Schroeder S, Krüger N, Herrler T, Erichsen S, Schiergens TS, Herrler G, Wu NH, Nitsche A, et al. SARS-CoV-2 cell entry depends on ACE2 and TMPRSS2 and is blocked by a clinically-proven protease inhibitor. Cell. 2020; 181(2): 271-80.

23. Fan CB, Li K, Ding YH, Lu LW, Wang JQ. ACE2 expression in kidney and testis may cause kidney and testis damage after 2019-nCoV infection. MedRxiv. 2020. doi: https://doi.org/10.1101/2020.02.12.20022418.

24. Rajapaksha IG, Gunarathne LS, Asadi K, Cunningham SC, Sharland A, Alexander IE, Angus PW, Herath CB. Liver-Targeted Angiotensin Converting Enzyme 2 Therapy Inhibits Chronic Biliary Fibrosis in Multiple Drug-Resistant Gene 2-Knockout Mice. Hepatol Commun. 2019; 3(12): 1656-73.

25. Minato T, Nirasawa S, Sato T, Yamaguchi T, Hoshizaki M, Inagaki T, Nakahara K, Yoshihashi T, Ozawa $\mathrm{R}$, Yokota S, et al. B38-CAP is a bacteria-derived ACE2-like enzyme that suppresses hypertension and cardiac dysfunction. Nat Commun. 2020; 11(1): 1058. 


\section{Tables}

Table 1

The association between the severity and myocardial injury indexes.

\begin{tabular}{|llll|}
\hline Parameters & Mild & Severe & Critically ill \\
\hline Cases, N & 213 & 90 & 52 \\
\hline CK (U/L) & $70.0(47.0,101.0)$ & $72.0(47.3,160.0)$ & $149(70.0,319.0) * * \# \#$ \\
\hline CKMB, N (\%) & $69(33.7)$ & $51(59.3)$ & $38(74.5)^{\star}$ \\
\hline LDH (U/L) & $222.0(186.0,272.0)$ & $299.0(226.0,370.0)^{\star *}$ & $442.0(277.5,630.3) * * \#$ \\
\hline AST (U/L) & $26.0(20.0,35.0)$ & $29.0(22.8,54.0) *$ & $49.0(35.0,80.0) * * \#$ \\
\hline $\begin{array}{l}\text { Myocardial injury } \\
\text { cases }(\%)\end{array}$ & $114(53.5)$ & $61(67.8) *$ & $45(86.5) * * \#$ \\
\hline
\end{tabular}


Table 2

The effects of demographic characteristics and complications on myocardial injury indexes.

\begin{tabular}{|c|c|c|c|c|c|}
\hline & Cases & CK (U/L) & $\begin{array}{l}\text { CKMB, N } \\
(\%)\end{array}$ & LDH (U/L) & AST (U/L) \\
\hline \multicolumn{6}{|l|}{ Gender } \\
\hline Female & 162 & $59.5(40.0,97.0)$ & $79(49.1)$ & $236.0(188.5,341.0)$ & $26.0(20.0,39.0)$ \\
\hline Male & 193 & $\underset{\star \star}{92.0}(59.5,153.5)$ & $76(41.5)$ & $254.0(201.5,337.5)$ & $30.0(23.0,47.0)$ \\
\hline \multicolumn{6}{|l|}{ Age } \\
\hline$\varangle 39$ & 96 & $75.5(41.5,101.5)$ & $19(19.8)$ & $209.0(172.0,261.0)$ & $23.0(19.0,29.0)$ \\
\hline $40-59$ & 144 & ${ }_{\star \star}^{69.0}(47.0,114.0)$ & $54(37.8)$ & ${ }_{* *}^{246.0}(200.3,327.5)$ & ${ }_{*}^{28.0}(22.0,45.0)$ \\
\hline$₫ 60$ & 115 & $\underset{\star \star \# \#}{99.0}(58.0,194.0)$ & $\begin{array}{l}82(71.3) \\
\star \star \# \#\end{array}$ & $\begin{array}{l}296.0(223.0,450.5) \\
\star \star \# \#\end{array}$ & $\begin{array}{l}36.0(23.0,57.5) \\
\star \star \# \#\end{array}$ \\
\hline \multicolumn{6}{|c|}{ Hypertension } \\
\hline Yes & 230 & $72.0(47.0,166.0)$ & $89(73.0)$ & $270.0(189.0,403.0)$ & $31.5(21.0,58.8)$ \\
\hline No & 125 & $77.0(50.0,122.0)$ & $69(30.7)$ & $239.0(195.8,312.8)$ & $27.0(21.0,39.0)$ \\
\hline \multicolumn{6}{|c|}{ Diabetes } \\
\hline Yes & 208 & $89.0(50.0,170.0)$ & $95(66.4)$ & $270.0(177.3,389.5)$ & $32.0(23.0,58.0)$ \\
\hline No & 147 & $72.0(49.0,113.8)$ & $63(30.9)$ & $237.0(197.0,306.0)$ & ${ }_{\star \star}^{26.0}(21.0,37.5)$ \\
\hline \multicolumn{6}{|l|}{$\begin{array}{l}\text { Heart } \\
\text { disease }\end{array}$} \\
\hline Yes & 20 & $78.0(47.0,184.0)$ & $12(63.2)$ & $346.0(223.0,480.0)$ & $35.0(21.0,71.0)$ \\
\hline No & 335 & $77.0(49.0,125.5)$ & $146(44.5)$ & $245.0(193.8,323.8)$ & $28.0(21.0,43.0)$ \\
\hline
\end{tabular}


Table 3

Multivariate logistic regression analysis risk factors of myocardial injury among COVID-19 patients.

\begin{tabular}{|lllll|}
\hline & $\boldsymbol{\beta}$ & Wald & P value & OR $(95 \% \mathrm{Cl})$ \\
\hline Male & 0.699 & 5.556 & 0.018 & $2.012(1.125,3.599)$ \\
\hline Age & -1.304 & 5.003 & 0.028 & $1.434(1.041,1.976)$ \\
\hline Hypertension & 1.222 & 7.814 & 0.005 & $3.393(1.441,7.989)$ \\
\hline Diabetes & 0.660 & 3.118 & 0.077 & $1.936(0.930,4.029)$ \\
\hline Heart disease & -0.016 & 0.000 & 0.984 & $0.984(0.202,4.788)$ \\
\hline
\end{tabular}

Table 4

The association between myocardial injury and death risk among COVID-19 patients.

\begin{tabular}{|c|c|c|c|c|}
\hline Myocardial injury & Cases & Death (\%) & $\mathrm{RR}(95 \% \mathrm{Cl})$ & $P$ value \\
\hline Yes & 220 & $33(15.0)$ & $8.625(2.107,35.305)$ & $\mathbb{\nabla} 0.001$ \\
\hline No & 115 & 2 (1.74) & 1 & - \\
\hline
\end{tabular}

Table 5

Myocardial injury indexes on admission and after discharge among COVID-19 patients.

\begin{tabular}{|c|c|c|c|c|c|c|}
\hline \multirow{2}{*}{$\begin{array}{l}\text { Myocardial } \\
\text { injury indexes }\end{array}$} & \multicolumn{3}{|c|}{ On admission $(\mathrm{N}=154)$} & \multicolumn{3}{|c|}{ Discharge $(N=150)$} \\
\hline & Median & $\begin{array}{l}\text { Below the } \\
\text { range, } \\
\mathrm{N}(\%)\end{array}$ & $\begin{array}{l}\text { Above } \\
\text { the range, } \\
N(\%)\end{array}$ & Median & $\begin{array}{l}\text { Below the } \\
\text { range, } \\
\mathrm{N}(\%)\end{array}$ & $\begin{array}{l}\text { Above the } \\
\text { range, } \\
N(\%)\end{array}$ \\
\hline CK (U/L) & $\begin{array}{l}65.0(44.0, \\
96.0)\end{array}$ & $45(29.8)$ & $5(3.3)$ & $\begin{array}{l}63.0(47.0, \\
82.0)\end{array}$ & $43(28.6)$ & 0 \\
\hline CKMB (U/L) & $\begin{array}{l}8.0(5.0 \\
13.0)\end{array}$ & 0 & $9(6.1)$ & $7.0(4.0,11.0)$ & 0 & $2(1.33) \#$ \\
\hline $\mathrm{LDH}(\mathrm{U} / \mathrm{L})$ & $\begin{array}{l}230.0 \\
(194.0 \\
278.0)\end{array}$ & $1(0.7)$ & $56(38.1)$ & $\begin{array}{l}203.0(176.0 \\
242.0) \star \star\end{array}$ & 0 & $25(16.7)$ \\
\hline AST (U/L) & $\begin{array}{l}24.0(20.0, \\
31.0)\end{array}$ & $23(15.0)$ & $19(12.3)$ & $\begin{array}{l}22.0(19.0 \\
30.0)\end{array}$ & $11(7.5)$ & $23(15.3)$ \\
\hline
\end{tabular}

